\title{
An Efficient Image Denoising Approach for the Recovery of Impulse Noise
}

\author{
S. Rajkumar ${ }^{* 1}$, G. Malathi ${ }^{2}$ \\ School of Computing Science and Engineering, VIT University, Chennai, India \\ ${ }^{*}$ Corresponding author, e-mail: rajkumar.srinivasan@vit.ac.in ${ }^{1}$, malathi.g@vit.ac.in ${ }^{2}$
}

\begin{abstract}
Image noise is one of the key issues in image processing applications today. The noise will affect the quality of the image and thus degrades the actual information of the image. Visual quality is the prerequisite for many imagery applications such as remote sensing. In recent years, the significance of noise assessment and the recovery of noisy images are increasing. The impulse noise is characterized by replacing a portion of an image's pixel values with random values Such noise can be introduced due to transmission errors. Accordingly, this paper focuses on the effect of visual quality of the image due to impulse noise during the transmission of images. In this paper, a hybrid statistical noise suppression technique has been developed for improving the quality of the impulse noisy color images. We further proved the performance of the proposed image enhancement scheme using the advanced performance metrics.
\end{abstract}

Keywords: image quality, impulse noise, visual quality, image denoising, image sharpen, image smoothing

\section{Introduction}

Noise is generally accumulated in to images during acquisition, transmission and compression. Image denoising is perhaps the most powerful operation of image processing and computer vision. The impulse noise is occurred due to the fault encountered in camera during the time of image transmission and reception, external disturbances in a booming natural environment, electromagnetic interferences, and timing problems in analog to digital conversion. The impulse noise is also called as spike noise, random noise or independent noise. Since the independent pixels in the image bears no relationship with the neighboring pixels.

The impulse noise is similar to salt and pepper noise with a slight difference. The impulse noise is characterized by random occurrences of bright and dark pixels and the impulse noise is characterized by random occurrences of bright dots. Mostly, the impulse noise is occurred in the electronic devices that transmits or receives the signal. The impulse noise arises from sudden spikes, which sometimes produces the annoying spots in the television signal.

The dust particles can also cause this type of noise. Uncertainties are the major impact of impulse noise. This fact makes image de-noising a difficult task. Understanding the uncertainties can enhance the quality of image de-noising. The algorithm in [1] uses two stage filtering algorithm in which the median value is computed twice and is used to remove the high density corrupted images. Amarjit Roy et.al. [2] proposed an artificial neural network (ANN) based fuzzy filter for removing the impulse burst. The ANN is used to classify the noise and signal from the image and based on the classification the fuzzy filtering is applied to adjust the noise and signal. In [3], an efficient method for detecting the impulse noise was developed. In this approach, once the noisy pixel is detected, the subsequent noise can be removed by various existing techniques such as switching median filters. The experimental result in [4] examines the contrast enhancement and tone mapping algorithms which applies to satellite images. In [5], an Additive White Gaussian Noise (AWGN) removal method was using the high resolution frequency concepts such as Non Harmonic Analysis (NHA) and is yielding good results in numerous applications such as image tomography and motion analysis. Enming Luo et. al [6] developed expectation-maximization (EM) adaptation method to denoise the document images. The above method yields good results when compared to existing denoising algorithm such as Expected Patch Log Likelihood (EPLL). The traditional noise removal methods cannot 
adequately remove the noise from the corrupted images. For this reason, Pok et. al [7], has been developed a separate class of nonlinear filters as based on homogeneous information for the removal of impulse noise. Tadahiro Azetsu et. al [8], use the rank order statistics filter to improve the quality of image which is corrupted due to impulse noise and is compared with traditional methods. The model in [9], is used to remove the Charge-Coupled device (CCD) noise and the experimental results proves the edge information as well. In [10], an optimal contrast enhancement algorithm is developed and is used to improve the contrast of the fog images. Bhat et.al in [11], is developed a hybrid approach that combines the back propagation neural network and adaptive median filtering in order to remove the salt and pepper noise and improve the image quality. In [12], a combined framework has been derived to restore the quality astronomy images. The above framework use the phase preserving technique, normalization method and Lucy debluring algorithm. A regularized minimization algorithm has illustrated by Fahmy [13], is used to minimize the error between noisy image and filtered image. Gonzalez-Hidalgo1 et.al [14], proposed a hybrid approach that inturn contain 2 stages. In the first stage, an impulse noise detector based on the fuzzy tophat transforms is used to detect the pixels which are likely to be composed by impulse noise. In the second level, the image is filtered using a regularized method that uses fuzzy open-close or fuzzy close-open sequences and is applied only to those selected infected pixels.

The technique in [15] aims to identifies the noisy pixels and replace the noisy pixels by the sparsity information. Xiao Kang et.al [16], presents a Novel Adaptive Switching Median (NASM) filter that effectively handles the salt and pepper noisy image and preserves the image details as well. Jinli Suo et al. can [17] remove highlight from high resolution images and is more complex compared to other existing approaches. A blind image restoration method in [18], is used to compute the noise variance and blur estimation without any prior knowledge. In [19], a novel Fuzzy Impulse noise Detection and Reduction Method (FIDRM) is proposed. The above filter has two phases: The first phase is the detection phase and the filtering phase. The first phase incorporates the fuzzy rules to identify whether the pixel is corrupted with impulse noise or not. The second phase is used for removing the impulse noise.

An efficient novel approach [20] was developed for de-focused images. The de-focus is occurred due to improper focus of camera lens. The model [20] can produce visually appealing HDR (High Dynamic Range) image. Two different exposure images are used as the inputs in the model. The shaken pixels between the images are identified. The blur pixels are removed and optimal quality image is obtained by integrating the large and small exposure images. In [21], the image quality is assessed by extracting the partial information from the image. The partial information is taken from the input image at the source point and the received image at the recipient side. An efficient quality assessment system was proposed and that provides more accurate and user friendly. In this method, certain features of the source image were taken through steerable pyramid decomposition algorithm and the image quality is measured by using the Reduced Reference (RR) method.

This paper has been organized in the following manner:

Section I deals with the introduction of the paper. Section 2 explains the motivation of the work. The research method-novel Hybrid Impulse Noise Removal (HINR) filter is illustrated in Section 3. The Section 4 discusses the results and analysis. Section 5 explains the conclusion and finally the future enhancement of our research method is explained in Section 6.

\section{Motivation of the Work}

Apart from the several techniques in image processing, the image denoising process is a rapidly evolving field with growing applications. Removal of noise from a signal is an important problem in image and signal processing. Noise is a practical problem raised in many systems such as satellite, medical and seismology.

The noise filtering is the form of image denoising and it becomes necessary to remove the noise and unwanted distortions such as impulse noise and mixed noise. Because of their wide applications, denoising is of great importance in signal and image processing. Since much effort was not taken, an initiative has been made to perform research in this area. 


\section{Research Method- Hybrid Impulse Noise Removal (HINR) Filter}

Earlier, the Order Statics Filters and Rank Order Rank Selection Filters were used to remove the impulse noise. But, those filters does not able to distinguish between minute details such as sharp edges and noise, also its performance becomes poor with increasing level of noise density. To eliminate all these drawbacks, in this paper we propose a Hybrid Impulse Noise Removal (HINR) filter. This filter is used to identify and handle high density impulse noisy color images and it covers wide range of samples of an image. It also preserves the fine details such as edges as well.

Step 1:

The novel HINR filter algorithm comprised of the following steps:

Convert the RGB input image in to grayscale using the relation:

Step 2:

Grayscale $=R^{\star} 0.21+G^{*} 0.72+B^{*} 0.01$

Let $n$ be the size of the window. Let us consider $n=9$. Since, the entire image is divided in to $3 X 3$ regions. Consider $f(x, y)$ is the center pixel. With a zero padding process, all the pixels are covered in this proposed algorithm. The mask $3 \times 3$ is traversed across the entire image start from $(0,0)$ co-ordinates to the entire size of the image.

a. if $f(x, y)<255$, then the 8 neighborhoods of the center pixel will be retained.

b. If $f(x, y)==255$, then the corresponding mask is subjected to the following conditions:

\section{Condition-1:}

If $(f(x-1, y+1)==255)$ and if $(f(x, y+1)==255)$ and if $(f(x+1, y+1)==255)$ and if $(f(x-1, y)==255)$ and if $(f(x+1, y)==255)$ and if $(f(x-1, y-1)==255)$ and if $(f(x, y-1)==255)$ and if $(f(x+1, y-1)==255)$ then

Compute the sum using the following relation:

Sum $=f(x-1, y+1)+f(x, y+1)+f(x+1, y+1)+f(x-1, y)+f(x+1, y)+f(x-1, y-1)+f(x, y-1)+f(x+1, y-1)$ then the average is to be computed and is replaced in the center pixel.

\section{Condition-2:}

Initiate Sum $=0$

If $(f(x-1, y+1) !=255)$ then

Sum $=\operatorname{Sum}+f(x-1, y+1)$

If $(f(x, y+1) !=255)$ then

Sum $=\operatorname{Sum}+f(x, y+1)$

If $(f(x+1, y+1) !=255)$ then

Sum $=\operatorname{Sum}+f(x+1, y+1)$

If $(f(x-1, y) !=255)$ then

Sum $=\operatorname{Sum}+f(x-1, y)$

If $(f(x+1, y) !=255)$ then

Sum $=\operatorname{Sum}+f(x+1, y)$

If $(f(x-1, y-1) !=255)$ then

Sum $=\operatorname{Sum}+f(x-1, y-1)$

If $(f(x, y-1) !=255)$ then

Sum $=\operatorname{Sum}+f(x, y-1)$

If $(f(x+1, y-1) !=255)$ then

Sum $=\operatorname{Sum}+f(x+1, y-1)$ then the average is to be computed and is replaced in the center pixel.

\section{Step 3:}

The output image is obtained from step (2) is the pure filtered image and thus the impulse noise is eliminated. But, the output image is imposed by the smoothing effect. Further, to avoid this smoothing effect, a sharpening filter is used.

Step 4:

The image obtained from step (2) is blurred (using box blur technique).

Subtract the blurred image from the obtained image through step (2). The resultant image contains only the enhanced edge information.

Step 5:

The image obtained from step (4) is added to the image obtained from step (2) to get the sharpened image and thus the smoothing effect in the filtered image is eliminated. 


\section{Results and Analysis}

The Image denoising process is vital part of many image and signal processing applications. In real time applications, the images are corrupted due to atmospheric turbulence, camera motion, aperture effects of the camera. Therefore an efficient image filter is required to further processing or analyzing the images. In our paper, a color image with impulse noise is considered as input and is shown in Fig.1. The size of the image is 256X256. As the preprocessing step, the input is image is converted in to grey scale and is shown in Fig.2. The results using existing fuzzy filter as explained in chapter 1 is shown in Fig.3. The filtered image with our proposed method is shown in Fig.4. The performance of our proposed method is assessed by using MSE, PSNR and structural content metrics. The results of the above measures are shown in Table 1.

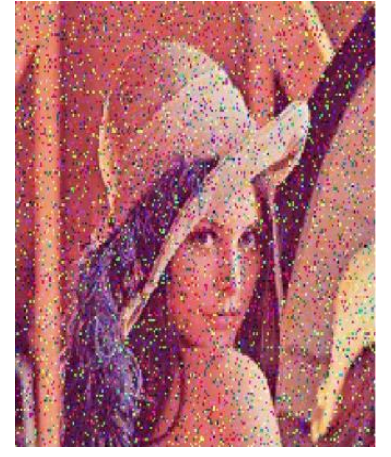

Figure 1. Impulse noisy Lena color image

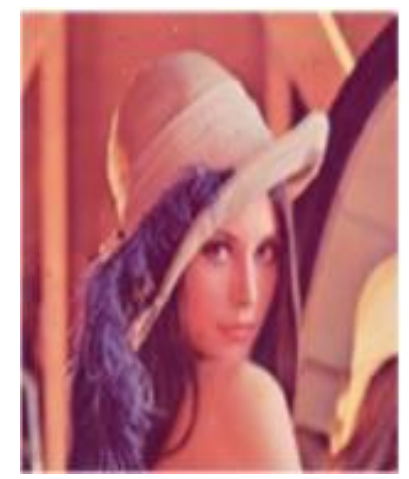

Figure 3. Result of Fuzzy Filtering Technique

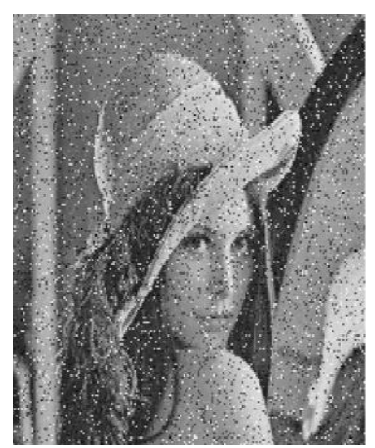

Figure 2. Impulse noisy grey scale image

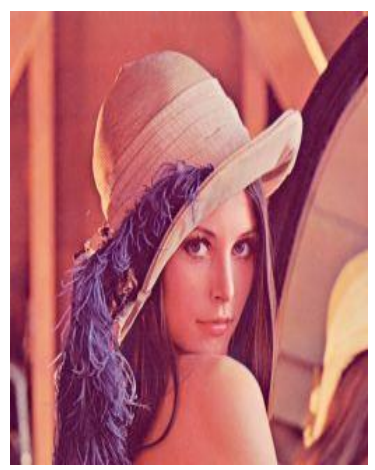

Figure 4. Result of our proposed HINR method

Table 1. MSE, PSNR and Structural Content Comparison for Colored Lena Image with

\begin{tabular}{cccc}
\multicolumn{4}{c}{ Fuzzy Filter } \\
\hline Filters & MSE & PSNR(db) & $\begin{array}{c}\text { Structural } \\
\text { Content }\end{array}$ \\
\hline $\begin{array}{c}\text { Fuzzy filter } \\
\begin{array}{c}\text { Proposed } \\
\text { HINR }\end{array}\end{array}$ & $\mathbf{3 1 8 . 8 2}$ & 21.95 & 0.37 \\
\hline
\end{tabular}

The Mean Square Error (MSE) metric is the dispersion metric and it can be used to quantify the image enhancement algorithm in which it is applied to removal of noise and blur. If the MSE value increases, then the image degradation increases. When MSE value reaches zero then pixel by pixel matching of images becomes perfect.

The MSE is calculated by using the following relation:

$$
\mathrm{MSE}=\frac{1}{\mathrm{MN}} \sum_{i=1}^{M} \sum_{j=1}^{N}\left((x(i, j)-y(i, j))^{2}\right.
$$


The Peak Signal to Noise Ratio (PSNR) is the most significant metric which is used to measure the visual quality of the restored image when it is corrupted due to noise and blur. Higher the value of PSNR, indicates higher the quality rate. The PSNR value is computed by using the following expression:

$$
\mathrm{PSNR}=10 \log _{10} \frac{\mathrm{MaxI}^{2}}{\mathrm{MSE}} \frac{\mathrm{MaxI}^{2}}{\mathrm{MSE}}
$$

The Structural Content (SC) deals with spatial arrangements of pixels in an image. This metric brings out the similarity between two images.

$\mathrm{SC}$ can be given as:

$$
\text { Structural Content }=\frac{\sum_{i=1}^{M} \sum_{j=1}^{N} x(i, j) X y(i, j)}{\sum_{i=1}^{M} \sum_{j=1}^{N}(x(i, j))^{2}}
$$

where $M$ is the number of pixels in horizontal direction, $N$ is the number of pixels in vertical direction, $x(i, j)$ is the noisy image at $i$ and $j$ co-ordinates and $y(i, j)$ is the filtered image at $i$ and $j$ co-ordinates.

\section{Conclusion}

The impulse noise is manifested by the random occurrences of pixel values. The impact of the impulse noise is the serious issue in image enhancement field. Therefore an effective, intuitive and robust denoising scheme is required to eliminate the impulse burst present in the real time images. Hence, in this paper, we proposed a filtering technique for removing the impulse noise in a color image. The results obtained using the proposed filter technique ensures noise free and quality of the image. It is shown that this filtering technique can provide an effective removal of impulse bursts. It preserves the edges of the image and covers wide range of samples. The quantitative analysis also proves that our HINR outperforms the existing Fuzzy filter. So, we can conclude that the resulting technique is a versatile and provides greatly improved efficiency while maintaining image quality and robustness.

\section{Future Enhancement}

The work can be extended to denoise any type of degradations such as Gaussian noise, Salt and pepper noise, Laplacian noise etc. This approach is to be modified and applied to the noises which affect the quality of satellite image

\section{Acknowledgement}

We wish to express our sincere gratitude and thanks to the reviewers for their constructive comments and valuable suggestions and we also highly pleased to our university for providing such a platform to carry out this research paper.

\section{References}

[1] Vikas Gupta, Rajesh Mahle, Umesh Gour. Image Noise Removal with Detail Preservation for Random Valued Impulse Noise. IEEE Int.Conf.on Engineering. 2013: 1-5.

[2] Amarjit Roy, Salam Shuleenda Devi, RH Laskar. Impulse Noise Removal from Gray Scale Images Based on ANN Classification Based Fuzzy Filter. In Proc. of IEEE Int. Conf. on Computational Intelligence and Networks. September 2016: 97-101.

[3] Isha Singh, Om Prakash Verma. High Density Impulse Noise Detection using Fuzzy C-means Algorithm. Defence Science Journal. January 2016; 66(1): 30-36.

[4] Jose-Luis Lisani, Julien Michel, Jean-Michel Morel, Ana Belén Petro, Catalina Sbert. An Inquiry on Contrast Enhancement Methods for Satellite Images. IEEE Trans. Geoscience and Remote Sensing, (This article has been accepted for inclusion in a future issue of this journal. Content is final as presented, with the exception of pagination) 2016.

[5] Fumitaka Hosotani, Yuya Inuzuka, Masaya Hasegawa, Shigeki Hirobayashi, Tadanobu Misawa. Image Denoising with Edge-Preserving and Segmentation Based on Mask NHA. December 2015; 24(12): 6025-6033. 
[6] Enming Luo, Student Member, IEEE, Stanley H. Chan, Member, IEEE, and Truong Q. Nguyen. Adaptive Image Denoising by Mixture Adaptation. IEEE Trans. Image Process. October 2016; 25(10): 4489-4503.

[7] G Pok, J Liu, AS. Nair. Selective Removal of Impulse Noise Based on Homogeneity Level Information. IEEE Trans. Image Process. January 2003; 12(1): 85-92.

[8] Tadahiro Azetsu, Noriaki Suetake, Eiji Uchino. Trilateral Filter Using Rank Order Information of Pixel Value for Mixed Gaussian and Impulsive Noise Removal. In Proc. of Int. Conf. IEEE 2013: 303-306.

[9] Hilda Faraji, W. James MacLean. CCD Noise Removal in Digital Images. IEEE Trans. Image Process. September 2006; 15(9): 2676-2685.

[10] Liu Bo, Xiong Qingguo. Inland River Image Defogging Based on Optimized Contrast Enhancement. In Proc. of IEEE Int. Conf. on Information Technology, Networking, Electronic and Automation Control Conference. Sep 2016: 145-150.

[11] Bhat Jasra, Aniqa Yaqoob, Sanjay Kumar Dubey. Removal of High Density Salt and Pepper Noise Using BPANN-Modified Median Filter Technique. In 6th IEEE Int. Conf. on Cloud System and Big Data Engineering. July 2016: 78-82.

[12] Z Al-Ameen, D Mohamad, M Shafry MR, G Sulong. Restoring Degraded Astronomy Images using a Combination of Denoising and Deblurring Techniques. International Journal of Signal Processing, Image Processing and Pattern Recognition (IJSIP). Mar 2012; 5(1): 1-12.

[13] Fahmy MF. A New Total Variation Based Image Denoising and Deblurring Technique. IEEE EUROCON. July. 2013; 21(4): 1669-1675.

[14] M González-Hidalgo1 S Massanet1 A Mir1 D. Ruiz-Aguileral. High-density Impulse Noise Removal Using Fuzzy Mathematical Morphology. In Proc. of Int. Conf. of the European Society for Fuzzy Logic and Technology (EUSFLAT 2013): 728-735.

[15] S Huang, J Zhu. Removal of Salt-and-pepper Noise Based on Compressed Sensing. IEEE Trans. Electronic Letters. August 2010; 46(17): 1198-1199.

[16] Xiao Kang, Wei Zhu, KeJie Li, Jing Jiang. A Novel Adaptive Switching Median Filter for Laser Image Based on Local Salt and Pepper Noise Density. Proc. of Int. Conf. IEEE 2011: 38-41.

[17] Jinli Suo, Dongsheng An, Xiangyang Ji, Haoqian Wang, Qionghai Dai. Fast and High Quality Highlight Removal from A Single Image. IEEE Trans. Image Process. August 2016.

[18] Chong Yi, Tetsuya Shimamura. A Blind Image Deconvolution Method Based on Noise Variance Estimation and Blur Type Reorganization. In Proc. of IEEE Int. Symposium on Intelligent Signal Processing and Communication Systems (ISPACS). December 2011.

[19] Stefan Schulte, Mike Nachtegael, Val'erie De Witte, Dietrich Van der Weken, Etienne E Kerre. $A$ Fuzzy Impulse Noise Detection and Reduction Method. IEEE Trans. Image Process. May 2006; 15(5).

[20] S Rajkumar, G Malathi. A Novel approach for the recovery of de-focused color Images. International Journal on Recent Researches in Science, Engineering and Technology (IJRRSET). May 2015; 3(5).

[21] S Rajkumar. An Advanced Technique for the Analysis of Image Quality Assessment for Distorted Images in the Field of Image Processing Application. In Proc. of conference in Karunya University, Coimbatore, India, Mar.2008: 84-88. 